\title{
Obtenção e comparação de texturas por processo de digitalização tridimensional
}

Leonardo Barili Brandi

Universidade Federal do Rio Grande do Sul

leonardo.barili@gmail.com

Renan Willian Leite Pereira

Universidade Federal do Rio Grande do Sul renan.pereira@ufrgs.br

Ana Maldonado

Universidade Federal do Rio Grande do Sul ana@navecomunica.com.br

Fábio Pinto da Silva

Universidade Federal do Rio Grande do Sul fabio.silva@ufrgs.br

Fábio Gonçalves Teixeira

Universidade Federal do Rio Grande do Sul fabiogt@ufrgs.br

Liane Roldo

Universidade Federal do Rio Grande do Sul liane.roldo@ufrgs.br

Resumo: Este estudo tem como objetivo utilizar a digitalização tridimensional a laser (por Holografia Conoscópica) e a digitalização por fotogrametria explorando o potencial do software 123D Catch, comparando e distinguindo os resultados. $O$ experimento foi realizado em três etapas: a digitalização tridimensional da superfície, onde foi selecionada a amostra - casca do melão, feito um projeto piloto de experimentação e realizados os processos de digitalização; comparação entre os processos, onde são comparados os processos de digitalização, em relação ao tempo de execução, recursos envolvidos, parâmetros, entre outros aspectos; comparação entre os resultados obtidos na digitalização da superfície, na qual é realizada uma análise macroscópica e de desvio no software Geomagic ${ }^{\circledR}$ Control 2014. Neste sentido, observou-se que o sistema de digitalização a laser (Digimill 3D) é preciso, tem maior resolução, verossímil e de maior custo no processo, porém são resultados esperados. Contudo, a digitalização com fotogrametria (123D Catch) pode 
ser suficiente, dependendo da qualidade necessária no projeto desenvolvido.

Palavras-chave: digitalização 3D, fotogrametria, 123D Catch, digitalização textura, design de superfície.

\begin{abstract}
This study aims to use the three-dimensional laser scanning (by conoscopic holography) and photogrammetry scanning exploring the potential of 123D Catch software, comparing and distinguishing the results. The experiment was performed in three steps: three-dimensional scan of the surfasse, where the sample was taken (melon peel), made a trial project and scan processes; comparison processes, where the scanning processes are compared in relation to the execution time, resources involved, parameters, among others; comparison between the results obtained from scanning the surface, in which a macroscopic and deviation analysis is performed in Geomagic ${ }^{\circledR}$ Control 2014 software. Accordingly, it was observed that the (Digimill 3D) laser scanning system is precise, has a higher resolution, it's believable and more expensive in the process, but results are expected. However, scanning with photogrammetry (123D Catch) may be sufficient, depending on the quality required in the project developed.
\end{abstract}

Keywords: three dimensional scanning, photogrammetry, 123D Catch, texture scanning, surface design.

\title{
1. INTRODUÇÃO
}

Novas tecnologias e ferramentas estão reduzindo o tempo de desenvolvimento e lançamento de novos produtos. Os consumidores têm maiores opções de escolha, e a cada dia surgem novidades. Um fabricante, que não seja capaz de se mover com rapidez suficiente nesse novo mundo de negócios, pode ficar seriamente comprometido. As estatísticas sobre sucesso dos negócios mostram uma clara participação crescente dos novos produtos. (BAXTER, 2011, p.17)

Uma dessas ferramentas é a Digitalização Tridimensional, que é utilizada na obtenção de dados computacionais de objetos físicos. Este processo é auxiliado por ferramentas computacionais que permitem grande precisão na captura dos dados dos detalhes do objeto, como a textura, vincos, curvaturas.

\subsection{Digitalização tridimensional}

É utilizada na obtenção de dados computacionais de objetos físicos. Este processo é auxiliado por ferramentas computacionais que permitem grande precisão na captura dos detalhes do objeto, como a textura, vincos, curvaturas. Com dados resultantes da digitalização é possível realizar: controle de qualidade; construção de moldes; personalização de objetos; entre outras inúmeras atividades que aceleram e tornam o processo de desenvolvimento de produtos mais flexível.

Nos últimos anos os sistemas de digitalização tridimensional foram significativamente melhorados. Métodos, técnicas de obtenção de dados e as ferramentas computacionais, evoluíram consideravelmente. 
As tecnologias de digitalização existentes podem ser classificadas como:

1. Com contato (necessitam de contato com a peça a ser escaneada)

Exemplos: CMM - Agulha com esfera na ponta; Caneta/Braço mecânico.

2. Sem contato (não necessitam de contato com a peça).

a) Ativos: emitem algum tipo de radiação.

Exemplos: Laser; Raio X; Luz Branca.

b) Passivos: não emitem radiação (com exceção da natural).

Exemplo: Fotogrametria.

Os sistemas utilizados estão se tornando populares para a indústria nacional, mas ainda necessitam de grandes investimentos, criando uma dificuldade de acesso a essa ferramenta na execução de projetos de baixo custo de desenvolvimento. Muitas vezes a necessidade do projeto é de redução de lead-time, e faz-se desnecessária a precisão na captura dos dados.

A fim de tornar esta tecnologia mais acessível, surgiram softwares de digitalização 3D como o 123D Catch, da desenvolvedora Autodesk ${ }^{\circledR}$ - distribuído gratuitamente -, em que são necessários menos recursos do que os métodos mais avançados de alta precisão existentes. $O$ software - que também tem sua versão para dispositivos móveis - realiza a digitalização 3D a partir de fotografias de câmeras comuns, processo conhecido como fotogrametria.

O estudo experimental em questão busca explorar o potencial do software 123D Catch, através da comparação entre dois métodos de digitalização distintos: digitalização à laser (por Holografia Conoscópica) e digitalização por fotogrametria, com a verificação de até que ponto se distinguem e comparação dos resultados.

Inicialmente, são descritos e comparados os dois processos; em seguida é realizado um processo experimental de digitalização de uma mesma superfície de textura tátil através dos dois processos; após a digitalização, realiza-se o processamento dos dados e tratamento dos modelos digitais, preparando-os para a comparação visual e de sobreposição das superfícies obtidas.

\subsubsection{Digitalização tridimensional a laser}

Optou-se em ter como referência a digitalização tridimensional a laser por esta ser mais precisa e rápida do que os sistemas com contato e também por ser considerada adequada para objetos com grande quantidade de detalhes.

"A digitalização 3D gera dados de saída que são processados geometricamente como coordenadas da superfície no espaço tridimensional $(x, y, z)$. Uma varredura superficial pode retornar milhares de pontos, de acordo com o tamanho da área analisada e o espaçamento entre pontos utilizado. Este conjunto de milhares de pontos é chamado de "nuvem de pontos" e após manipulação computacional permite gerar curvas, malhas, superfícies e sólidos tridimensionais compatíveis com sistemas CAD/CAE/CAM" (BATISTA, 2009). O funcionamento desse sistema é descrito no item 2.4.1.

\subsubsection{Digitalização tridimensional por fotogrametria}

A Fotogrametria pode ser definida como a ciência aplicada, a técnica e a arte de extrair de fotografias dimensionais, formas, e posições de objetos que estão nela contidos. 
Segundo, ASP(1966), fotogrametria é a arte, ciência e tecnologia de obter informações de confiança sobre objetos e do meio ambiente com o uso de processos de registro, medições e interpretações das imagens fotográficas e padrões de energia eletromagnética registrados.

A fotogrametria pode ser utilizada em registros arquitetônicos, registros arqueológicos, na obtenção de modelos digitais de terrenos, medições industriais, engenharia reversa e mapeamento topográfico. Para a digitalização tridimensional, o sistema baseia-se na sobreposição de uma sequência de fotografias através da identificação de elementos comuns nas mesmas.

\subsection{Processo experimental}

O experimento foi realizado em três etapas descritas a seguir: digitalização tridimensional da superfície, onde é selecionada a amostra, feito um projeto piloto de experimentação e realizados os processos de digitalização; comparação entre os processos, onde são comparados os processos de digitalização, em relação ao tempo de execução, recursos envolvidos, parâmetros, entre outros aspectos; 3) comparação entre os resultados obtidos na digitalização da superfície, na qual é realizada uma análise macroscópica e de desvio no software Geomagic ${ }^{\circledR}$ Control 2014.

\section{DIGITALIZAÇÃO TRIDIMENSIONAL DA SUPERFÍCIE TEXTURIZADA}

\subsection{Escolha da superfície}

A superfície escolhida para verificar o processo de digitalização pelo 123D Catch é textura da casca de um melão, conhecido no Brasil como Melão Orange (Cucumis melo L.). Sua casca possui rendilhamento corticoso (Figura 1) com relevo naturalmente irregular de aproximadamente $0,4 \mathrm{~mm}$ de profundidade quando seco.

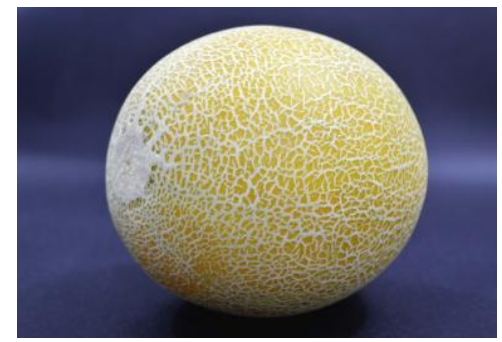

Figura 1 - Melão Orange

Os critérios para a escolha desta partiram da busca por uma textura de leve percepção tátil, que pudesse ser encontrada no ambiente cotidiano, que desafiasse o potencial do software e que tivesse escala reduzida a ponto de explorar o outro viés da fotogrametria, comumente utilizada para digitalização de superfície terrestre, terrenos, edificações, interiores, entre outros artefatos de grandes dimensões.

Além disso, buscou-se uma textura que pudesse ser parametrizada e aplicada no desenvolvimento de produtos. A textura pode ser percebida muitas vezes como um fator diferencial, apesar de ser mais uma variável dentro do processo de concepção formal e raramente ser desenvolvida como um alvo por si só, mas sim como consequência das características intrínsecas do projeto e sua tecnologia (MINUZZI, 2001). Considerando que cada material possui uma textura diferente segundo sua natureza - proveniente da natureza ou resultado de processos de fabricação - e segundo sua composição física, pode-se ainda trabalhar com uma variedade notável de 
possibilidades de texturização, das quais o designer deve estar ciente para que possam ser postas a serviço de uma expressão visual e tátil.

\subsection{Preparação da amostra}

O fruto foi cortado longitudinalmente com uma faca e foram removidas as sementes e a polpa, restando somente aparte externa casca. Para que a forma do material orgânico se mantivesse estática entre um processo de digitalização e outro, as amostras permaneceram em secagem por 15 dias para atingirem o estado de equilíbrio de umidade e massa constante, na atmosfera condicionadora de temperatura $23 \pm 1^{\circ} \mathrm{C}$ e umidade relativa $50 \pm 2 \%$

Com escalímetro e tesoura, foram medidos e recortados quadrados de $3 \mathrm{~cm} \times 3$ $\mathrm{cm}$. Em seguida estas amostras ficaram prensadas em chapas de MDF por 5 dias, para que mantivessem uniformidade e facilitassem o posicionamento das lentes utilizadas na captura quanto à distância focal (Figura 2).
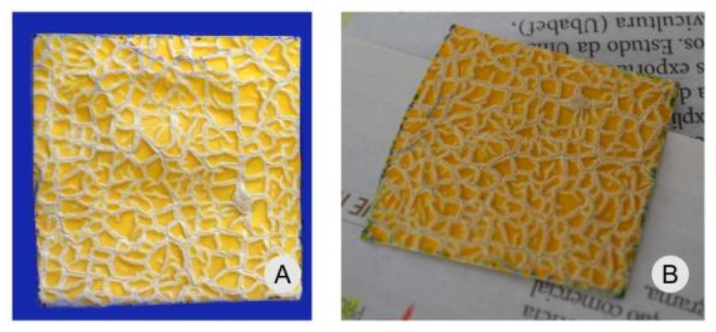

Figura 2 - Amostra selecionada e recortada em 30x30mm (A); Amostra prensada após 5 dias (B).

\subsection{Piloto}

Antes do experimento, foi realizado um experimento piloto a fim de verificar o processo de captura e digitalização do 123D Catch: equipamentos necessários, recursos envolvidos, extensão e compatibilidade de arquivos, qualidade da digitalização gerada, tipo de malha exportada e exploração das funções do software. Para isso, foram seguidas à risca as recomendações do site do desenvolvedor - que estão descritas no desenvolvimento dos processos, nos itens a seguir. A importância deste experimento preliminar demonstrou-se no mapeamento do processo, onde já puderam ser levantados meios de otimizar cada atividade.

\subsection{Processos de digitalização}

Nos itens a seguir são descritos os dois processos - digitalização a laser e digitalização por fotogrametria - utilizados para se obter uma malha triangular da mesma amostra de casca de Melão Orange. Os processos são claramente distintos, porém, tem-se a digitalização a laser como referência de resultado esperado. Portanto, quanto mais a digitalização por fotogrametria se aproximar, na comparação das malhas de textura geradas, mais satisfatório se torna o resultado.

\subsubsection{Digitalização tridimensional a laser(por holografia conoscópica)}

O equipamento utilizado para a digitalização a laser é o Digimill 3D, da marca Tecnodrill ${ }^{\circledR}$, instalado nas dependências do Laboratório de Design e Seleção de Materiais da UFRGS. Além deste, também foram utilizados: um microcomputador com o software Geomagic ${ }^{\circledR}$ Studio v.10.

O sistema funciona através de holografia conoscópica, onde é utilizado um cabeçote de laser pontual que realiza a varredura do objeto ponto a ponto, podendo 
adquirir até 1.000 pontos por segundo. O sistema de captura pode estar equipado com lentes de $25 \mathrm{~mm}$ a $250 \mathrm{~mm}$, que permite ajustar a melhor relação entre precisão, faixa de captura, distância do objeto e tempo de varrimento.

Com a amostra e equipamento preparados, seguiu-se o fluxograma de atividades (Figura 3) proposto por Silva (2006):

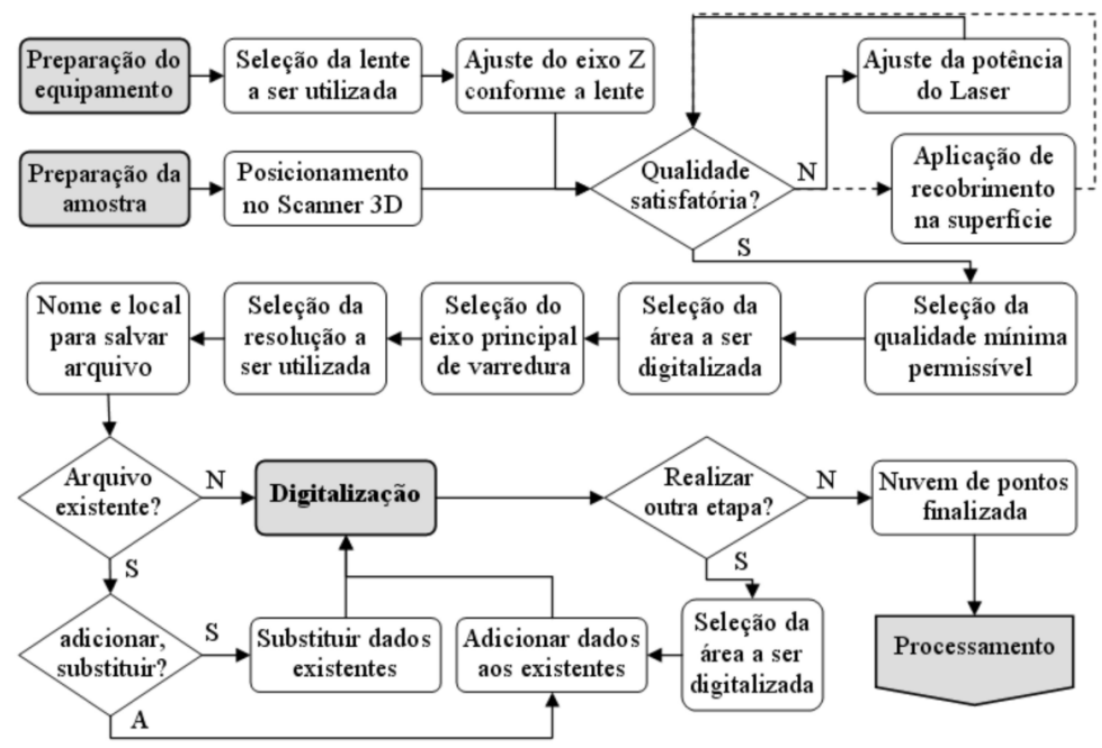

Figura 3 - Fluxograma do processo de digitalização a laser. Fonte: Silva, 2006.

Após escolha da lente $(50 \mathrm{~mm})$ delimitação da área, escolha do eixo de varredura, ajuste focal e da potencial do laser foram determinada a resolução entre pontos de 0,1 mm - considerado suficiente para a textura envolvida.

Definidos os parâmetros, o equipamento realiza a varredura de pontos, gerando a nuvem de pontos - arquivo de extensão .TXT - posteriormente processada e manipulada em softwares específicos para esta função.

O software utilizado para o processamento e manipulação foi o Geomagic $^{\circledR}$ Studio versão 10, da desenvolvedora 3D Systems. Primeiramente, a nuvem de pontos foi filtrada pelo comando de redução de ruído (eliminou pontos discrepantes da nuvem). Em seguida, transformada em uma malha de triângulos regulares (327.161 triângulos) e gerada uma superfície, exportada em extensão de arquivo OBJ identificada como extensão compatível entre os dois processos (Figura 4 e Figura 5).
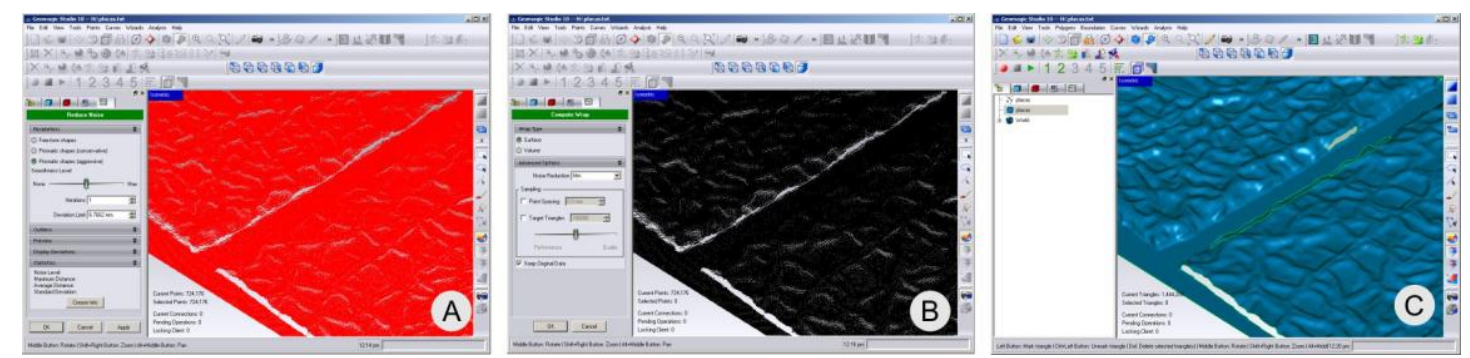

Figura 4 - Processamento da nuvem de pontos no Geomagic Studio 10: redução de ruídos (A), transformação em malha da triângulos (B) e superfície gerada (C). 


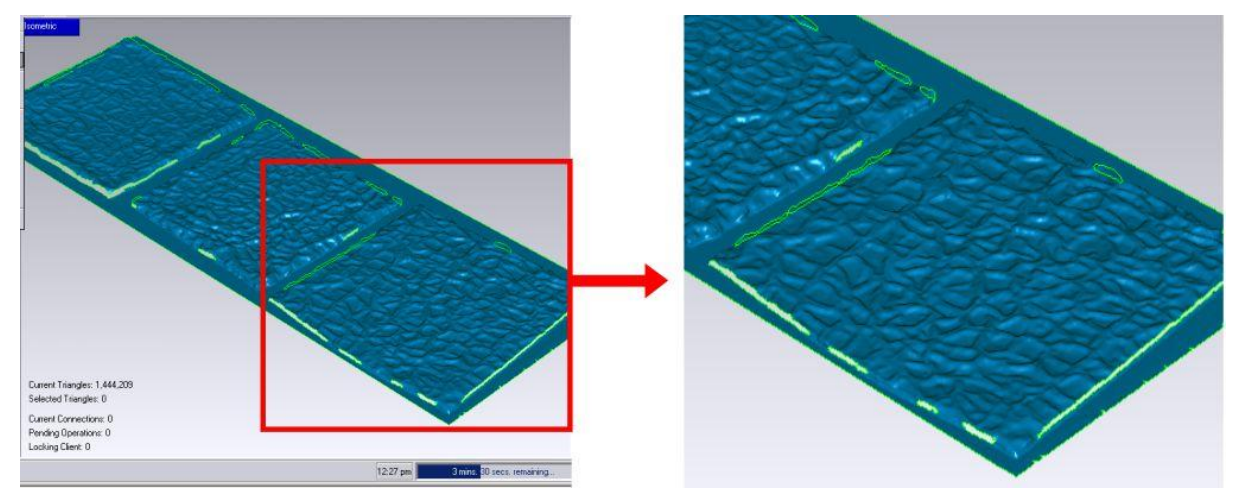

Figura 5 - Resultado da superfície digitalizada a laser.

Foram colocadas 3 amostras para digitalização - para optar pela mais uniforme posteriormente - e o tempo de digitalização das mesmas foi de $19 \mathrm{~min}$. O tempo de preparação do equipamento foi aproximadamente de $15 \mathrm{~min}$. e o tempo de processamento e manipulação dos dados foi de $36 \mathrm{~min}$. Acredita-se que à medida que este processo seja realizado outras vezes, a experiência prática reduzirá este tempo de preparação e manipulação. Quanto ao processamento, quanto melhor o hardware para executar o software, menor o tempo investido.

\subsubsection{Digitalização tridimensional por fotogrametria: 123D Catch}

Os equipamentos utilizados para a digitalização por fotogrametria foram: máquina fotográfica SLR Nikon ${ }^{\circledR}$ D3200, com lente $18-55 \mathrm{~mm}$, uma mesa, e microcomputador com o software 123D Catch versão offline instalado e acesso à internet.

O processo foi guiado pelas instruções e dicas fornecidas no site da empresa desenvolvedora do software (http://www.123dapp.com/howto/catch) somados aos conhecimentos adquiridos no experimento piloto desta ferramenta.

A preparação do equipamento começa com o ajuste de zoom e foco da lente, para mantê-los constantes e centralizados no objeto. Assim como na captura a laser, reflexos, brilhos e transparências no objeto interferem na captura, portanto é necessário ajustar a iluminação de modo que fique constante e estável, além de desligar o flash da máquina fotográfica. Pode ser utilizado luz solar, desde que as fotos sejam tiradas com o mínimo intervalo de tempo e não crie muitas sombras.

Para a preparação da amostra deve-se posicioná-la de modo que o fotógrafo possa se movimentar livremente ao redor dele - recomenda-se coloca-la no centro de uma mesa - para que todas as partes do objeto possam estar acessíveis pela fotografia. A superfície de fundo também deve ser preparada para conter os pontos de referências que conectam uma foto à outra. Recomenda-se utilizar folhas de papel com texto e/ou símbolos impressos, com cores contrastantes (Figura 6).

Quanto às fotografias, deve-se manter o foco no objeto, centralizado na fotografia e tirar fotografias sequenciais ao redor de toda amostra, por pelo menos 2 ângulos diferentes (sugestão de $30^{\circ}$ e $60^{\circ}$ em relação à base do modelo) realizando uma sobreposição de pelo menos $50 \%$ de uma fotografia para a outra. O número máximo de fotografias que pode ser enviado para o processamento é de 70 fotos. Superfícies modulares ou idênticas não conseguem ser captadas, bem como modelos simétricos ou ambíguos, e não é recomendado utilizar alta exposição ou baixa exposição. 


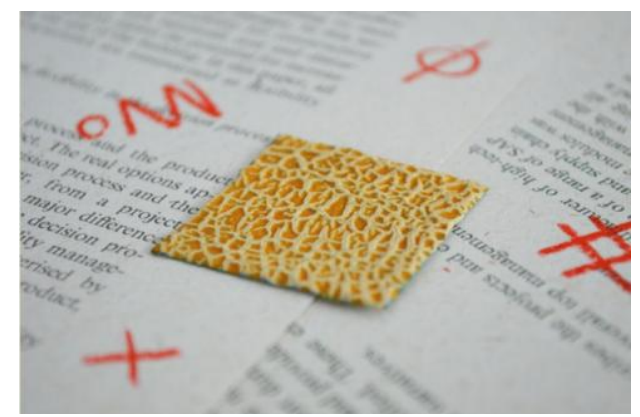

Figura 6 - Exemplo de uma das fotos geradas para o processamento.

Para este estudo, selecionou-se a amostra e foram tiradas entre 45 e 50 fotografias, com distância focal de $45 \mathrm{~mm}$, dimensão de $3008 \times 2000$ px por foto e 300 dpi de resolução. $O$ total de imagens gerou $111 \mathrm{Mb}$ de arquivos para o processamento. Em seguida, essas fotos foram manipuladas com software de edição de bitmap para reduzir seu tamanho ( $80 \mathrm{Mb}$ ), a fim de facilitar o upload dos arquivos, porém contrabalanceando com uma resolução suficiente para o processamento. Além disso, o contraste da fotografia original foi ajustado para aumentar a saturação e destaque das linhas.

O processamento das fotografias no 123D Catch ocorre num sistema de processamento em nuvem, ou seja, as fotos são enviadas pela internet para um servidor que acessa computadores de alta capacidade de processamento e reenvia o modelo digital na qualidade de malha selecionada. Em seguida, pode-se manipular a digitalização, com ferramentas básicas de seleção, órbita, visualização da fotografia sobre a malha e cotagem.

Ao contrário do processo de digitalização a laser através do sistema Digimill 3D, os parâmetros da fotogrametria pelo 123D Catch são, essencialmente, automáticos: não é possível escolher resolução da captura, por exemplo. Os parâmetros são itens passíveis de alteração a favor de melhores resultados na digitalização, no caso do 123D Catch os parâmetros controláveis ficam por conta dos equipamentos envolvidos na obtenção das fotografias e escolha da qualidade da malha exportada - que são "Mobile" (baixa), "Standard" (média) e "Maximum" (alta).

Independente da escolha da qualidade, que varia o número e tamanho dos triângulos na malha, a mesma é formada por triângulos irregulares e o software a exporta em arquivo de extensão OBJ. A malha exportada pelo 123D Catch utilizada nesse estudo foi a de qualidade alta (Maximum) formada por 41.115 triângulos.

Referentes à qualidade da fotografia pode-se alterar a resolução da câmera, contraste da imagem, lente, aproximação do objeto (zoom); número de fotografias (embora o software selecione automaticamente as fotografias passíveis de uso, a quantidade de fotos tiradas ao redor do modelo fornece mais opções para essa filtragem). 
O fluxograma do processo apresenta-se na Figura 7:

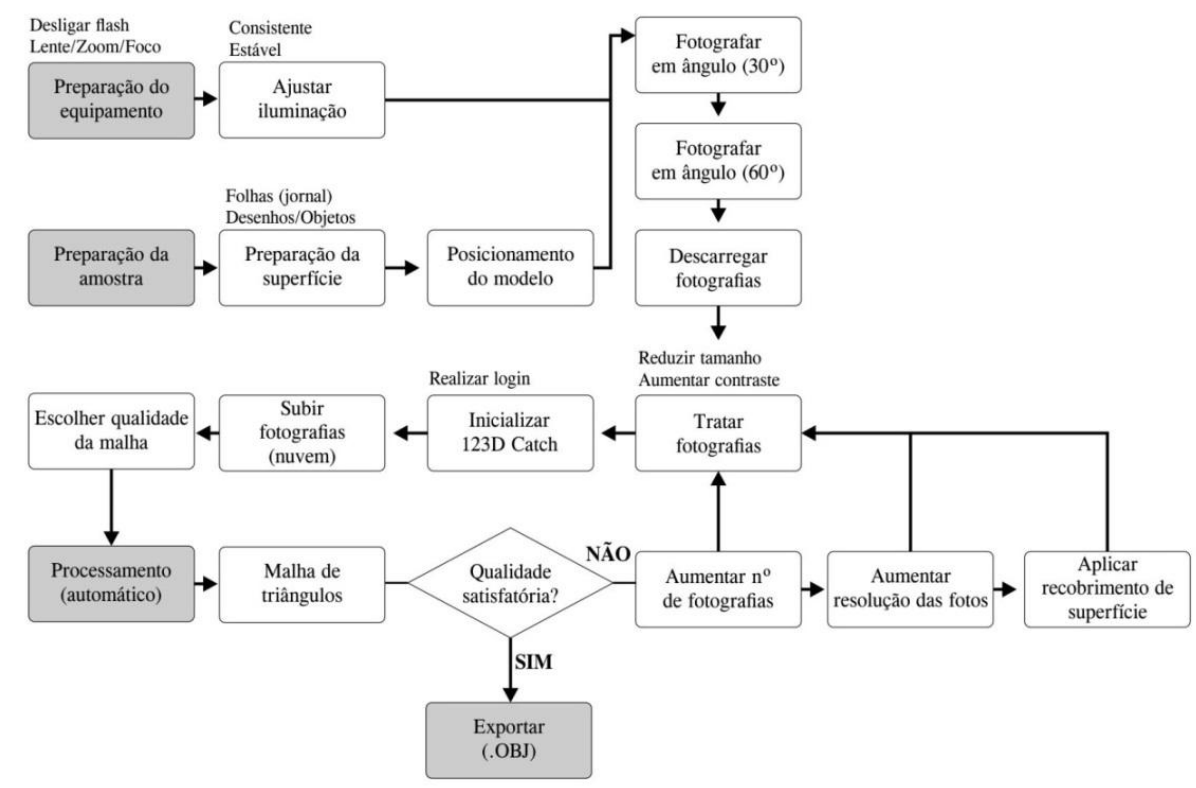

Figura 7 - Processo de digitalização por fotogrametria através do software 123D Catch.

O tempo de preparação do equipamento e da amostra foi de aproximadamente 15 min. considerando os ajustes na máquina fotográfica, a iluminação, preparação da superfície e posicionamento do modelo. Para a realização das fotografias e tratamento foram cronometrados aproximadamente $20 \mathrm{~min}$. Já para o envio e processamento dos dados para se obter a malha ocorreu em $12 \mathrm{~min}$. sob a velocidade média de upload de $30 \mathrm{kbps}$.

Da mesma forma que na digitalização a laser, a experiência prática e o hardware utilizado podem causar variações grandes na medida de tempo, bem como a velocidade da internet pra realização de upload das fotos e download da malha de triângulos.

\section{Análise comparativa entre os processos}

\subsection{Peculiaridades dos processos}

A digitalização a laser é um processo mais automatizado. $O$ operador é exigido no ajuste do equipamento e preparação da amostra, enquanto na fotogrametria ele realiza os mesmo procedimentos e ainda executa e manipula as fotografias sequenciais.

\subsection{Parâmetros}

O Digimill 3D possui parâmetros realmente controláveis na pré digitalização e pré processamento. Aumentar a resolução para captura de mais pontos ou ajustar a potência do laser são alguns exemplos. Os parâmetros "controláveis" do 123D Catch dependem muito mais dos ajustes no equipamento, na superfície e manipulação das fotografias, onde são experimentadas diversas técnicas para otimizar a sobreposição das fotografias, sem saber a lógica que o software executará sobre elas.

\subsection{Custos}


Em estudos comparativos relacionados (RIVEIRO, 2013; GONZÁLES-JORGE, 2012), entre os mesmos processos de digitalização, torna-se clara a vantagem da fotogrametria sobre a questão de recursos envolvidos, principalmente equipamento. Segundo Gonzáles-Jorge (2012), "as duas técnicas demonstram suas capacidades metrológicas e tolerâncias necessárias, contudo a fotogrametria aparece como uma técnica aconselhável [...] exige investimento econômico mais baixo e oferece boa portabilidade, apesar de requerer mais experiência do operador [...]"(tradução nossa).

Pode-se constatar que a aquisição do sistema para fotogrametria tem valores muito abaixo do sistema a laser, embora a taxa de precisão seja inferior. Segundo Silva (2006), ainda existem outros processos mais acessíveis que o 123D Catch (Figura 8):

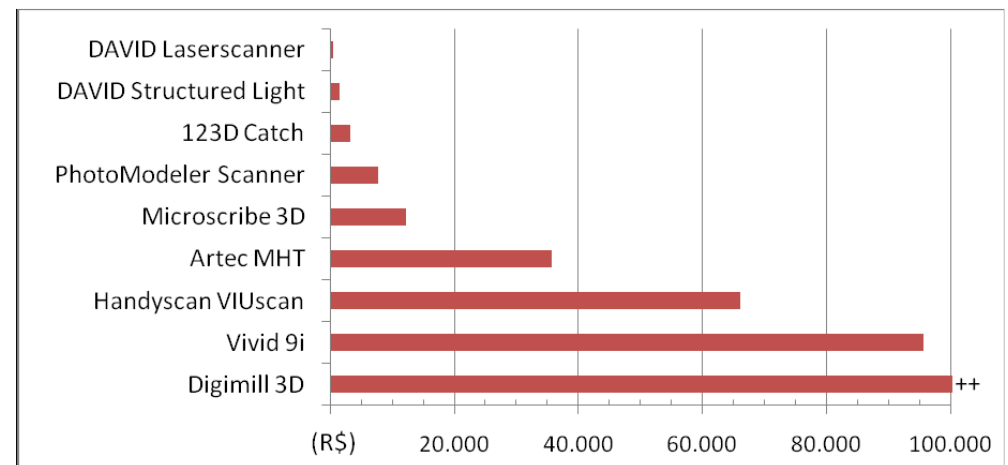

Figura 8 - Relação de custos estimados entre diferentes sistemas de digitalização. Fonte: SILVA, 2006.

\subsection{Tempo}

Os tempos totais dos processos, da preparação à malha gerada, foram de aproximadamente $47 \mathrm{~min}$. para o sistema 123D Catch e de1 h e10 min. para o Digimill 3D. Contudo, a falta de experiência do operador pode mudar significativamente estes valores. Neste caso, os dois processos só haviam sido realizados pelo operador no experimento piloto feito com cada um.

\subsection{Processamento}

O 123D Catch necessitou de cerca de $111 \mathrm{Mb}$ de arquivos de imagens, para enviá-los via internet, processar em nuvem e receber a malha em arquivo de extensão OBJ de 5,559 Mb; o outro sistema gerou uma nuvem de pontos em arquivo extensão TXT de cerca de $18 \mathrm{Mb}$ que, após processamento no software Geomagic ${ }^{\circledR}$ Studio, gerou uma malha em arquivo de extensão OBJ de 33,445 Mb.

\section{Análise comparativa DOS RESULTADOS}

\subsection{Preparação da superfície e comparação macroscópica}

Para comparação macroscópica utilizou-se o software Rhinoceros ${ }^{\circledR}$ versão 5 . Ao importar os arquivos oriundos do 123D Catch e Geomagic ${ }^{\circledR}$ Studio para dentro do ambiente virtual do software ocorreu um erro de escala que foi corrigido através de comandos provenientes do programa (Figura 9). 


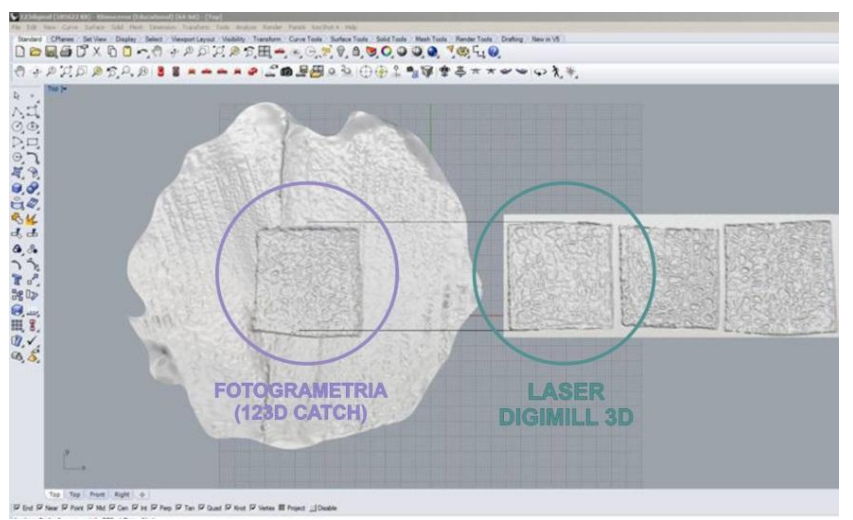

Figura 9 - Ajuste de escala e padronização da superfície para comparação.

Definiram-se cores para diferenciação das malhas, verde para a digitalização a laser (sistema Digimill 3D) e lilás para fotogrametria (sistema 123D Catch). Para melhor manipulação dos modelos matemáticos foi realizado um recorte para que ambos os modelos ficassem com as mesmas dimensões.

Em uma análise macroscópica notou-se que a textura do modelo matemático do 123D Catch é menos profunda e definida que a do Digimill 3D. Uma das causas dessa discrepância é atrelada à construção da malha exportada pelo 123D Catch: a malha de triângulos irregulares, neste caso, desfavorece o modelo digital. A única vantagem é tornar o arquivo mais leve para a manipulação (Figura 10). A malha do 123D Catch é formada por 41.115 triângulos irregulares, enquanto a outra possui 327.161 triângulos regulares.
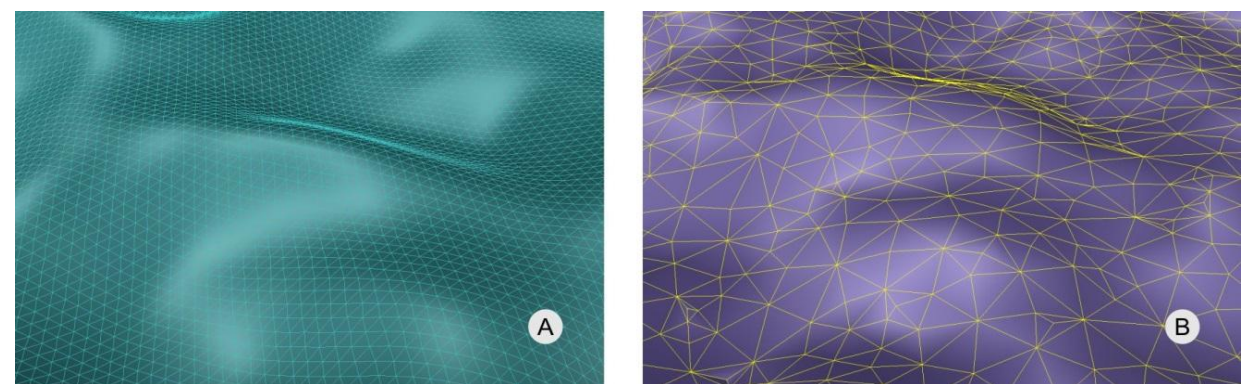

Figura 10 - Triangulação das malhas: na digitalização a laser foi possível gerar uma malha de triângulos regulares (A), enquanto o 123D Catch exporta somente malha de triângulos irregulares (B).

\subsection{Comparação por sobreposição (comparação 3D)}

Sobre as mesmas amostras digitalizadas, realizou-se a comparação por sobreposição através do software Geomagic ${ }^{\circledR}$ Control 2014 (versão trial). O software ajusta automaticamente através de algoritmos a posição de "encaixe" entre os dois modelos. O desvio padrão entre as amostras foi de 0,0992 $\mathrm{mm}$, tendo desvios máximos de 1,5720 $\mathrm{mm}$ positivos e 1,3169 $\mathrm{mm}$ negativos (Figura 11). Esses resultados não são considerados satisfatórios se levada em conta a precisão dos modelos. 


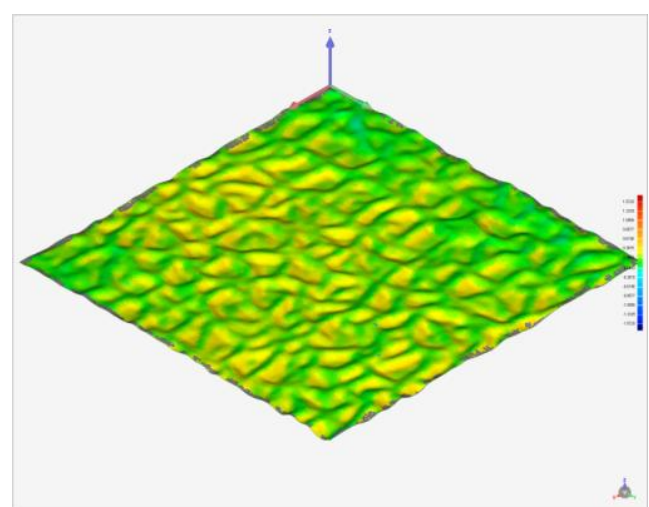

Figura 11 - Gráfico de comparação 3D dos modelos digitais sobrepostos.

\section{CONCLUSÃO}

A digitalização a laser (Digimill 3D) é precisa, tem maior resolução, verossímil e de maior custo no processo, porém são resultados esperados. Contudo, a digitalização com fotogrametria (123D Catch) pode ser suficiente, dependendo da qualidade necessária no projeto desenvolvido.

A manipulação dos dados gerados pelo 123D Catch não requer altos investimentos em equipamentos (hardware). Concordamos com Riveiro (2013), que aponta: "a principal vantagem da técnica de fotogrametria vem dos baixos custos do sistema" (tradução nossa).

Algumas possibilidades do software gratuito 123D Catch foram testadas e, apesar das limitações de ajustes de parâmetros, foi possível melhorar a captura dos dados, otimizando o contraste das fotos e aumentando o número de fotografias. 0 tempo de processamento dos dados pode ser otimizado pela prática do operador e velocidade da internet, tornando o processamento em nuvem outra vantagem.

Sobre as amostras, sugere-se que, em trabalhos futuros, as amostras sejam escaneadas através do laser com alta resolução e usinadas em um material sólido inorgânico. Assim, seria possível comparar as texturas deste sólido, sem perder a referência por quaisquer possíveis deformações da amostra orgânica.

Além disso, propõe-se que seja estudada a possibilidade de realizar a digitalização de superfícies por fotogrametria utilizando imagens de estereoscópio. Um dos testes preliminares para a comparação deste trabalho envolveu o uso de fotografias sequenciais de estereoscópio e o resultado não foi satisfatório.

Contudo, com os parâmetros identificados posteriormente, acredita-se que seja possível obter melhores resultados, sendo necessária uma adaptação no equipamento para tirar fotos de 2 ângulos diferentes, em 360 graus, com iluminação constante.

\section{REFERÊNCIAS}

AMERICAN SOCIETY OF PHOTOGRAMMETRY(ASP). Manual of Photogrammetry.1966, $1220 \mathrm{p}$.

BADIRU, A. I.; RAMOS, A. R. D.; SANTOS, L. R. D.; SILVA, G. J. F.; BEPPLER, M. Utilização de fotogrametria terrestre para modelagem 3D de imóveis na cidade de João Pessoa - PB. IV Simpósio Brasileiro de Ciências Geodésicas e Tecnológicas de Geoinformação. Recife - Pe, 06-09 de Maio de 2012, p. 1-6. 
GONZÁLES-JORGE, H.; RIVEIRO, B.; ARIAS, P.; ARMESTO, J. Photogrammetry and laser scanner technology applied to length measurements in car testing laboratories. In: Measurement. v. 45, 2012, p. $354-363$.

MUNARI, B. Design e comunicação visual. Rio de Janeiro: Edições 70, 1968.

RIVEIRO, B.; GONZÁLES-JORGE, H.; VARELA, M.; JAUREGUI, D. V.; Validation of terrestrial laser scanning and photogrammetry techniques for the measurement of vertical under clearance and beam geometry in structural inspection of bridges. In: Measurement. v. 46, 2013, p. $784-794$.

SILVA, F. P. O uso da digitalização tridimensional a laser no desenvolvimento e caracterização de texturas aplicadas ao design de produtos. 2006. Dissertação (mestrado), Universidade Federal do Rio Grande do Sul, Departamento de Engenharia de Materiais, Porto Alegre.

\section{AGRADECIMENTOS}

Os autores agradecem ao Programa de Pós Graduação em Design da UFRGS, ao Laboratório de Design e Seleção de Materiais pela estrutura, equipamentos e suporte - especialmente a técnica do laboratório Márcia C. Machado. 